\title{
VISUALIZAÇÃO DE AMBIENTES IMERSIVOS: UMA PERSPECTIVA CONSIDERANDO WEB VIEWERS
}

Fabrício Herpich, PPGI, UFSM, fabricio.herpich@gmail.com

Andressa Falcade, PPGI, UFSM, andressafalcade@gmail.com

Aliane Loureiro Krassmann, PPGI, UFSM, alkrassmann@gmail.com

Luiz Evandro Garcia da Silva, PPGI, UFSM, luizevandro.silva@gmail.com

Rafaela Ribeiro Jardim, PPGI, UFSM, rafa.rjardim@gmail.com

Josmar Nuernberg, PPGTER, UFSM, josmarsm@gmail.com

Roseclea Duarte Medina, PPGI, UFSM, roseclea.medina@gmail.com

\begin{abstract}
Resumo. Ambientes imersivos são tecnologias amplamente utilizadas no âmbito educacional devido as potencialidades pedagógicas, entretanto sua utilização requer a instalação de um software para acesso à interface gráfica, o qual possui papel importante na correta visualização do ambiente. Neste sentido, este estudo realiza uma análise qualitativa sobre tais ferramentas, denominadas viewers, com enfoque nas tecnologias para a plataforma web, que tornam-se uma alternativa, apresentando as suas principais características e funcionalidades de acordo com o contexto educacional.
\end{abstract}

Palavras-chave: Ambientes Imersivos, Mundos Virtuais, Web Viewers.

\section{VISUALIZATION OF IMMERSIVE ENVIRONMENTS: A PERSPECTIVE CONSIDERING WEB VIEWERS}

\begin{abstract}
Immersive environments are technologies widely used in the education sector due the yours pedagogical potential, but its use requires the installation of software to access graphical interface, which plays an important role in the correct environment view. Thus, this study performs a qualitative analysis of these tools, called viewers, focusing on technologies for the web platform. Thus, this study performs a qualitative analysis of these tools, called viewers, focusing on technologies for the web platform, becoming an alternative, presenting the main features and functionalities according to the educational context.
\end{abstract}

Keywords: Immersive Environments, Virtual Worlds, Web Viewers.

\section{INTRODUÇÃO}

As Tecnologias de Informação e Comunicação (TIC) estão trazendo novidades para o uso educacional, como jogos sérios, ambientes virtuais de aprendizagem e mundos virtuais (MV). Para Herpich et al. (2014) há uma necessidade de incorporar novas metodologias no processo de ensino, visando estender a construção do conhecimento para além do ambiente presencial de sala de aula.

Os mundos virtuais são aplicações de simulação para criação de espaço virtual em três dimensões totalmente imersivo onde os usuários podem explorar, interagir e colaborar uns com os outros (Farooq e Glauert, 2011). Os metaversos são aplicações que necessitam de um servidor para sua hospedagem e funcionamento, e de um viewer, o qual faz a conversão do código para a visualização gráfica e deve ser instalado no 
computador, demandando tempo, conhecimento e manutenção por parte do usuário, situação que pode inibir a sua utilização.

Nesta perspectiva, os viewers para a web surgem como uma alternativa com grande potencial, pois permitem inclusive a inserção de arquiteturas baseadas em cloud computing, possibilitando o compartilhamento em rede, sob demanda de recursos, de plataformas, de servidores e de aplicações. Essa característica reduz a necessidade de infraestrutura computacional ao usuário final, facilitando a utilização e permitindo a qualquer pessoa o acesso, independente do dispositivo escolhido, pois exige um mínimo ou nenhuma instalação de software adicional.

O objetivo deste estudo decorre da importância de identificar viewers desenvolvidos para serem utilizados na web, com o intuito de dar continuidade ao trabalho de Nunes et al. (2013) sobre viewers para ambientes virtuais imersivos e suas características. Porém, o enfoque é diferenciado, uma vez que verifica as formas de visualização de MVs através da internet, e a efetividade dessas novas abordagens. Para tanto, são apresentados alguns viewers para a web, bem como suas principais características e funcionalidades que podem auxiliar na escolha dos mesmos.

\section{AMBIENTES VIRTUAIS IMERSIVOS E A EDUCAÇÃO}

É notável o crescimento dos ambientes virtuais imersivos em instituições acadêmicas, por ser uma tecnologia inovadora que permite o acesso remoto a atividades e conteúdos de sala de aula, onde os estudantes se tornam mais participativos, reativos, abertos e criativos (Loureiro e Bettencourt, 2010). Os autores Medina (2004) e Voss et al. (2013) discursam ainda, que uma aprendizagem gerada por experiências pessoais e com interação de outros participantes é mais produtiva, consolidada e dinâmica.

Estes ambientes são espaços tridimensionais baseados em computador, que podem ser explorados em primeira ou em terceira pessoa, através de uma representação gráfica chamada avatar (Nelson e Erlanderson, 2012). Para Albuquerque e Velho (2003), os metaversos foram projetados para fornecer aos usuários uma experiência que parece natural ou real e não mediada, com o objetivo de dar-lhes um sentido de presencialidade em ambientes para compartilhamento de vivências com outros usuários.

O desenvolvimento destes ambientes acontece através de plataformas específicas que, segundo Voss (2014), disponibilizam um espaço virtual compartilhado por vários usuários, que permitem a produção de conteúdos a partir das necessidades dos usuários finais e auxiliam no desenvolvimento de ambientes complexos. Como exemplos destas plataformas podem-se citar o Second Life (2014), o OpenSimulator (2014) e o OpenWonderland (2014).

Para Reis et al. (2013), essas plataformas estão sendo muito utilizadas no desenvolvimento de atividades educacionais, pois possibilitam a simulação de tarefas de aprendizagem em diferentes áreas do conhecimento, gerando o aprendizado através da experiência. Estes ambientes também favorecem a aprendizagem, já que facilitam o processamento, apresentação e compreensão da informação, tornando o estudante protagonista da sua própria aprendizagem, ofertando métodos variados para a construção do conhecimento (Nájera et al., 2011).

No contexto deste trabalho os MVs são considerados como espaços tridimensionais onde o estudante pode transitar e vivenciar experiências em um ambiente altamente interativo (Orgaz et al., 2012), sendo essa tecnologia uma alternativa às práticas educativas, de forma mais flexível com relação ao espaço/tempo, auxiliando o processo de aprendizagem em instituições acadêmicas, pois agregam 
praticidade na realização de tarefas que transcendem o contexto tradicional de sala de aula (Voss et al., 2013).

\section{VIEWERS E SUAS CARACTERÍSTICAS}

Os viewers são ferramentas que o usuário instala e configura para realizar o acesso ao ambiente virtual imersivo. Segundo Nunes et al. (2013), são softwares responsáveis pelo acesso remoto ao metaverso mantendo uma conexão para que os usuários interajam com os objetos e elementos contidos nele a partir de seus avatares.

Dessa maneira, Voss (2014) acrescenta que além de prover a visualização do mundo virtual, o viewer também fornece o suporte para a importação e exportação de objetos e demais recursos, porém, cada visualizador possui características próprias que podem influenciar na sua utilização final. Para o autor, a escolha do viewer é fundamental devido às limitações impostas por cada software, o que pode gerar problemas, e.g. restrição na importação de objetos e visualização limitada, dificultando assim, a visualização do metaverso. Portanto, o viewer deve estar alinhado aos objetivos e as expectativas dos alunos e ao contexto pedagógico, atendendo suas necessidades.

\subsection{Web Viewers}

Os viewers de forma resumida, têm o intuito de potencializar a navegação dos usuários no mundo virtual. Além disso, também são incorporados outros diversos aspectos relacionados à Interação Humano-Computador (IHC), sustentando fundamentos de interface, usabilidade, interação, design, entre outros.

Nesta perspectiva, os viewers específicos para a plataforma web surgem como alternativas viáveis, tanto no que tange a facilidade de acesso proporcionada aos usuários, os quais não necessitam da instalação e configuração de softwares robustos em seus computadores, quanto também no que se refere à disponibilidade de hardware avançado e sistemas operacionais suportados pela ferramenta, provendo assim, o acesso de qualquer lugar, momento e plataforma, necessitando apenas de conexão banda larga.

A escolha dos visualizadores utilizados nos testes, teve como referência a pesquisa de Falcade et al. (2014), que analisou, inicialmente, vinte ferramentas e apresentou seis delas como sendo web viewers. Os autores dessa pesquisa tiveram como base os trabalhos de Nunes et al. (2014), Voss et al. (2013), Ávila et al. (2012) e a página HyperGrid (2014). Portanto, os web viewers selecionados para compor este estudo foram:

O TipoDean disponibiliza regiões de livre acesso para teste, visualizadas na Internet, por meio de um plugin Unity 3D. As vantagens desse viewer são a facilidade de acesso, mesmo com um hardware menos avançado e com menor largura de banda; a possível integração com redes sociais; e a alta escalabilidade (Tipodean, 2014).

O Sandbox é um projeto de aprendizagem distribuída, com sua construção em HTML5 dispensando a instalação de plugins, baseado na web. É construído em Virtual World Framework, que possui um site de demonstração onde o usuário pode ter uma noção de seu funcionamento, podendo ainda ser configurado em seu próprio servidor (VW Sandbox, 2014). Este viewer possibilita ao usuário, criar o seu próprio mundo conforme suas necessidades (e.g. reunião, palestra e jogos), bem como alterá-lo utilizando a ferramenta de criação de objetos.

O 3D Xplorer possui uma gama de serviços (e.g. eventos virtuais, websites 3D, live meeting, web conferencing, salas de aula e exposições virtuais), sendo um viewer 3D interativo, totalmente web, ou seja, não requer nenhum download ou instalação e 
funciona com qualquer navegador em qualquer computador (3D Xplorer, 2014). Já o Cube3 é um projeto voltado para os clientes do entretenimento e mundo empresarial, trabalhando com web viewers e jogos Web3D. Os seus visualizadores são personalizados e podem ser acessados por navegador web, seja em computador ou smartphone, projetados para trabalhar com tecnologias multiusuário 3D e Java, utilizando o plugin web Unity 3D (Cube3, 2014).

O ReactionGrid é uma empresa de criação de simuladores 3D multiusuário e de viewers, com foco no ensino, uso comercial e entretenimento. A plataforma Jibe é carrochefe da ReactionGrid para a construção e implantação de ambientes 3D na web e em dispositivos móveis, que utiliza o plugin Unity 3D (Reaction Grid, 2014). Já o PixieViewer trata-se de um visualizador web sem a instalação de qualquer software ou plugin, além de ser responsivo e permitir acesso através de dispositivos móveis. Foi desenvolvido em HTML5 utilizando WebGL, possui funcionalidades básicas, como criação de objetos, texturização e chat (Voss et al., 2013).

\section{MÉTODO DE PESQUISA}

A fim de discutir e analisar as características e as funcionalidades de cada web viewer apresentado neste estudo, baseando-se em uma metodologia qualitativa e tecnológica, foram estabelecidas as seguintes etapas: a) Formulação do objetivo do estudo e de revisão bibliográfica; b) Pesquisa por web viewers existentes para os testes; c) Formulação dos parâmetros de avaliação; d) Aplicação e documentação dos testes seguindo os parâmetros pré-estabelecidos; e) Análise e discussão dos resultados obtidos.

Para os testes em computador foram utilizados os browsers Chrome, Internet Explorer (IE) e Firefox, apontados como os três mais acessados no mundo, no período entre Janeiro e Julho de 2014 na pesquisa da StatCounter (2014). Já os testes em dispositivos móveis, foram conduzidos no navegador Chrome, pelos aparelhos definidos na Tabela 1, lembrando que o objetivo não foi a avaliação dos dispositivos, mas sim, a visualização das regiões disponibilizadas para teste nos web viewers. Assim, todos os dispositivos utilizaram a mesma conexão de banda larga.

Tabela 1. Especificação dos dispositivos

\begin{tabular}{c|c|c|c}
\hline $\begin{array}{c}\text { Dispositivos / } \\
\text { Especificações }\end{array}$ & Galaxy Duos 2 & Galaxy S3 & Tablet 10.1 \\
\hline Fabricante & Samsung & Samsung & Samsung \\
\hline Modelo & GT-S7582 & GT-19300 & GT-N8000 \\
\hline Versão & Android 4.2 & Android 4.0 & Android 4.1.2 \\
\hline Tamanho da tela & $4.0 ”$ & $4.8 ”$ & 10,1 \\
\hline Memória & 4 GB & 16 GB & $2 \mathrm{~GB}$ \\
\hline Processador & Dual Core 1.2 Ghz & Quad-Core $1.4 \mathrm{Ghz}$ & Quad Core $1.4 \mathrm{GHz}$ \\
\hline
\end{tabular}

Para a realização dos testes foram escolhidos os MVs disponibilizados em cada viewer. Com o Tipodean foi acessada a região Rutgers University. Já no Cube3, foi visualizada a região Demo Unit. No viewer SandBox realizou-se o teste na região Desert Village. A região Jibe (Milspec World) foi a testada no ReactionGrid. No viewer 3DXplorer foi visualizada a região Auditorium2 e no PixieViewer foi acessada a região default, visto que a ferramenta está em teste e não permite a seleção de outras regiões.

\section{DESENVOLVIMENTO DO ESTUDO}

A partir dos atributos apontados como relevantes para viewers nos trabalhos de Amaral et al. (2013) e Nunes et al. (2013), buscou-se adaptar algumas características para esta 
pesquisa, elencando 10 critérios que devem ser contemplados por viewers para ambientes virtuais imersivos. Na Tabela 2 são abordados os critérios e as particularidades de cada visualizador e como respostas possíveis definiu-se: Sim, Parcial e Não, às quais foram atribuídos os valores 10, 5 e 0 , respectivamente, baseando-se na escala Likert (Likert, 1932) para avaliação posterior dos resultados.

Tabela 2. Comparativo entre a ferramentas de visualização para ambientes imersivos

\begin{tabular}{|c|c|c|c|c|c|c|}
\hline Viewer / Critério & TipoDean & 3DXplorer & SandBox & Cube3 & ReactionGrid & PixieViewer \\
\hline Compatibilidade com MVs & $\operatorname{Sim}^{1}$ & Parcial & Parcial & $\operatorname{Sim}^{1}$ & $\operatorname{Sim}^{1}$ & Parcial \\
\hline $\begin{array}{c}\text { Região (ões) com Livre } \\
\text { acesso } \\
\end{array}$ & Sim & Sim & Sim & Sim & Sim & Sim \\
\hline Oferece Serviços & Sim & Não & Sim & Não & Não & Sim \\
\hline $\begin{array}{c}\text { Integração com Redes } \\
\text { Sociais }\end{array}$ & $\operatorname{Sim}^{1}$ & Sim & Não & Não & Não & Não \\
\hline $\begin{array}{c}\text { Correta visualização do } \\
\text { ambiente }\end{array}$ & Parcial & Parcial & Parcial & Parcial & Parcial & Parcial \\
\hline Acesso em navegadores & Sim & Sim & Parcial & Parcial & Parcial & Sim \\
\hline Dispositivos móveis & Não & Sim & Parcial & Não & Não & Parcial \\
\hline Importação de Objetos & Não & Não & Sim & Não & Não & Sim \\
\hline Criação de objetos & Não & Não & Sim & Não & Não & Sim \\
\hline $\begin{array}{l}\text { Requer download ou } \\
\text { instalação de plugin }\end{array}$ & Sim & Parcial & Não & Sim & Sim & Não \\
\hline
\end{tabular}

${ }^{1}$ Informação disponível no site oficial (Tipodean, 2014), (Cube3, 2014) e (Reactiongrid, 2014).

Como podem ser visualizados na Tabela 2, todos os viewers fornecem as regiões de livre acesso, disponíveis para testes, contudo apresentam dificuldades de visualização e interação em algumas regiões. Por exemplo, na região do Tipodean o avatar não consegue subir escadas e entrar em prédios, sendo que esse fator ocorre, também com o viewer ReactionGrid e PixieViewer. No Tipodean ocorre também, a falta de interação com os objetos, pois o avatar tem, somente, a possibilidade de se locomover pela região. Isso também acontece no ReactionGrid e Cube3. Acredita-se que este fato ocorra por ser uma versão de teste, somente para visualização do produto.

Já na região do viewer ReactionGrid há uma inconsistência no grid: em alguns locais o avatar consegue atravessar objetos e paredes e em outros não, o que também ocorre no visualizador 3DXplorer. No ReactionGrid algumas paredes não permanecem visíveis o tempo todo, dependendo da posição do avatar, o que acontece, também, com o SandBox. Essa característica pode ser observada nas Figuras 1 e 2, onde o avatar está visualizando o mesmo prédio, sob duas perspectivas, interna e externa.

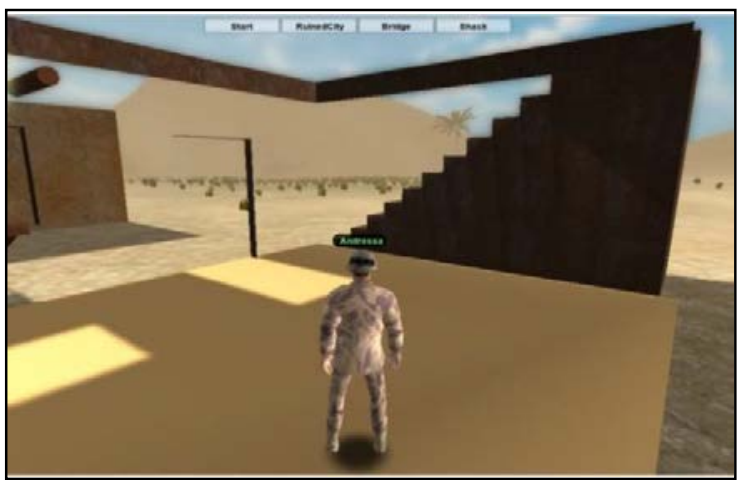

Figura 1. Visão interna do prédio

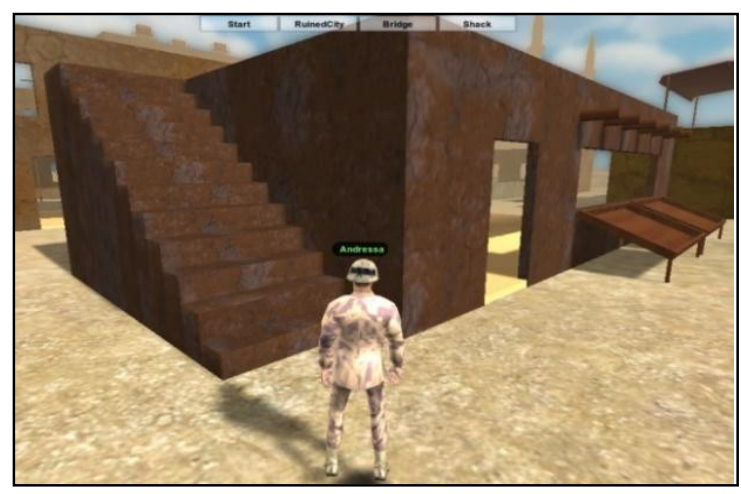

Figura 2.Visão externa do prédio no ReactionGrid 
As características como boa visualização do ambiente e de interação com objetos podem ser um diferencial na hora da escolha de um web viewer, pois quanto mais completo o cenário e quanto maior o sensação de imersão do usuário dentro do MV melhor será a sua impressão para uma utilização futura, o que só acontece quando o ambiente é mostrado na íntegra e com detalhes.

Os viewers 3DXplorer, SandBox e PixieViewer, fornecem câmeras que permitem a visualização do ambiente através de diferentes perspectivas. Além disso, fornecem interação com outros avatares por meio de chat e voz, e interação com os objetos, como clicar e realizar diversas ações. O SandBox possibilita alterar a linguagem e o modo de visualização de tela, de normal para estéreo (duplicação de telas) e modo Tablet (tela cheia) o que permite ao usuário escolher a forma que mais atende as suas necessidades. Já o 3DXplorer, por ser um viewer de web conference, permite envio de arquivo para apresentação de slides, compartilhamento da área de trabalho, transmissão de vídeos e o uso de apontadores de laser. Apesar das limitações apontadas, os viewers ReactionGrid, PixieViewer e 3D Xplorer apresentaram boa aparência gráfica, maior quantidade de informação e melhor interação no ambiente.

Em relação a possibilidade de acesso a outros MV foi atribuído o valor "sim" para os viewers que acessam mais de um metaverso. São eles o Tipodean, o Cube3 e o ReactionGrid, que disponibilizam o acesso ao OpenSim e ao Second Life. Em contraponto foi atribuído "parcial" aos viewers que permitem acesso apenas ao seu próprio MV. São eles o 3DXplorer, o SandBox e o PixieViewer. Quando há opção de escolher o MV a que se tem preferência, sendo o Second Life, o OpenSim, ou outro, a possibilidade de escolha desse viewer se torna maior, devido à flexibilidade e adaptabilidade as exigências do usuário.

Outra característica que deve ser percebida é a disponibilidade de serviços adicionais, encontrados no Tipodean, PixieViewer e Sandbox. O Tipodean realiza a conversão do MV para a plataforma web com o Unity 3D e a vantagem desse serviço é a possibilidade de portabilizar um MV, disponibilizando-o na web, preservando todas as características. Já o PixieViewer oferece o serviço de impressão de objetos 3D diretamente do viewer, não sendo necessária a instalação de um software, necessitando apenas da impressora 3D. O Sandbox permite a criação de novas regiões de teste passíveis de alterações. Essa funcionalidade permite que os usuários conheçam as limitações e possibilidades de criação da ferramenta, ideal para quem deseja construir seu próprio MV.

A criação e importação de objetos diretamente do viewer é uma característica que está presente nos visualizadores convencionais mais utilizados, como o FireStorm e o Imprudence. Essa característica está presente nos web viewers SandBox e PixieViewer, que permitem a criação de formas pré-definidas, e. g. círculos, retângulos, linhas, cilindros, quadrados, entre outros. Além disso, permitem modificá-los, alterando sua posição e tamanho. Quanto à importação, o Sandbox aceita o upload de modelos tridimensionais em arquivo "zip", contendo as extensões, .dae .fbx, .obj, .3ds ou .skp. Além disso, oferece uma loja de repositórios que permite ao usuário comprar e baixar objetos e texturas para o viewer. Essa característica é fundamental, por permitir caracterizar e modificar a região diretamente no ambiente gráfico, sem a necessidade de conhecimento específico em programação, necessária na formulação dos mundos virtuais através das plataformas de desenvolvimento para desktop.

No último item da tabela sobre a requisição de download ou instalação de plugin, os valores referentes às respostas "sim” e "não" foram invertidos, visto que são 
fatores negativos neste estudo (o que se busca com o web viewer é a flexibilidade de um serviço que não necessita de ferramentas e de mecanismos adicionais para funcionar, ou seja, totalmente portável). Os testes mostraram que os viewers Tipodean, Cube3 e ReactionGrid necessitam da instalação do plugin Unity 3D. Esse fator, além de ser negativo para o quesito facilidade de acesso, também impediu os mesmos de serem visualizados nos dispositivos móveis Galaxy Duos 2, Galaxy S3 e Tablet 10.1, pois os aparelhos não suportam o plugin. Já o 3D Xplorer possibilita o acesso de duas formas, através de HTML5 (Modo Padrão) e também por meio do plugin Java (Modo 3D imersivo para usuários avançados). Nos dispositivos móveis testados, ele só foi acessado pelo HTML5, pois os mesmos não tinham suporte ao plugin Java.

Os viewers SandBox, e PixieViewer não exigem instalação de software ou plugin. Porém, o Sandbox não permitiu a completa visualização através dos dispositivos móveis testados, não terminando o carregamento da região, apresentando um erro de login. O PixieViewer não pode ser visualizado através dos dispositivos móveis Galaxy Duos 2 e Galaxy S3, apesar do site oficial apresentar que ele pode ser acessado por qualquer aparelho. Contudo, ele foi acessado através do Tablet 10.1, pois apresenta maior número de núcleos de processamento, já que o PixieViewer exige alta capacidade para a renderização (Voss et al., 2013).

No teste com os navegadores, os viewers Tipodean, 3DXplorer e PixieViewer podem ser acessados normalmente pelos três browsers (Firefox, Chrome e Internet Explorer). Já os viewers ReactionGrid e Cube3 não permitem ser acessados pelo navegador IE, por ser incompatível com o plugin Unity 3D, ou seja, mesmo o plugin já estando instalado no computador, o browser não o reconhece.

O viewer SandBox, apesar de ter sido acessado pelos três navegadores, apresentou diferenças de visualização entre eles. No IE o SandBox teve suas cores verdes modificadas para o azul, como mostra as figuras 3 e 4. Já no Firefox a movimentação do avatar foi impedida.

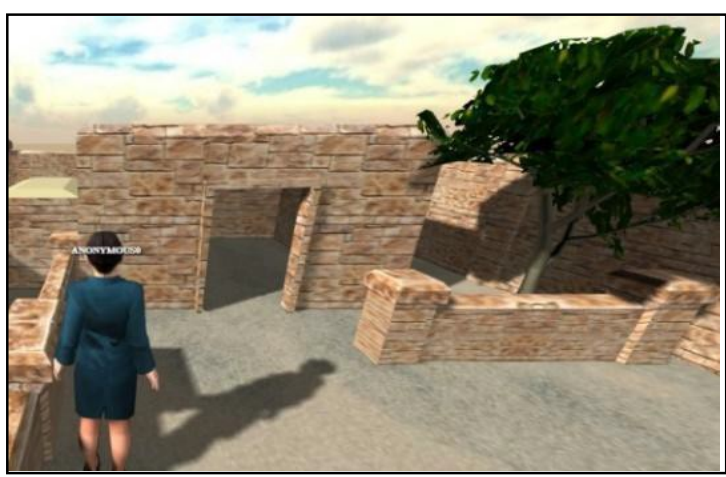

Figura 3. Sandbox no Browser Chrome

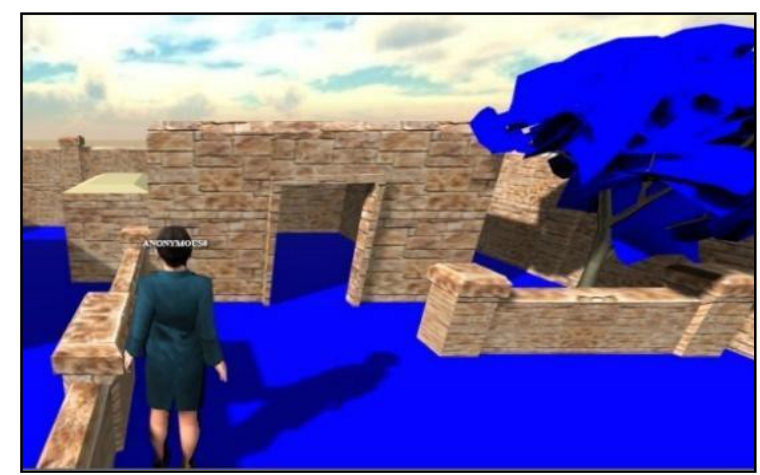

Figura 4. Sandbox no Browser IE

Com base na pesquisa de StatCounter (2014), sabe-se que há uma diferenciação na escolha do navegador, seja por preferência ou por funcionalidade. Portanto, o viewer que se adapta ao maior número de browsers, tem maior possibilidade de ser escolhido para utilização. Em relação à integração dos viewers com as redes sociais, apenas o Tipodean e o 3DXplorer permitem que os usuários utilizem suas contas de Twitter, Google e Facebook para acessá-los. Essa vantagem facilita a utilização do ambiente, pois agiliza o acesso, resgatando os dados já informados na conta da rede social. 


\section{DISCUSSÃO DOS RESULTADOS}

Para melhor compreender a eficiência de cada viewer, as respostas da Tabela 2 foram transformadas em valores. Esses valores foram somados, sabendo-se que a menor nota possível seria zero (0) e a maior nota possível de ser alcançada seria cem (100), como demonstra o Gráfico 1.

Com a análise do gráfico, pode-se observar que o PixieViewer obteve a maior pontuação (75) seguido do viewer SandBox (70). Isso ocorre porque ambos os viewers oferecem um amplo número de funcionalidades, entre elas, importação e criação de objetos, além de disponibilizarem serviços e oferecer boa portabilidade.

Já os viewers Tipodean e 3D Xplorer obtiveram uma pontuação mediana (55). O Tipodean requer a instalação adicional do plugin Unity 3D e o 3D Xplorer pode ser visualizado, tanto por HTML5 quanto pelo plugin Java Player. Ambos deixam a desejar no quesito gráfico, como mencionado na seção 5 . Além disso, não permitem a criação e importação de objetos. Entre as vantagens destacam-se o serviço adicional que o Tipodean oferece (Conversão para acesso via Unity 3D) e o acesso via dispositivos móveis, possibilitado pelo 3DXplorer através do HTML5.

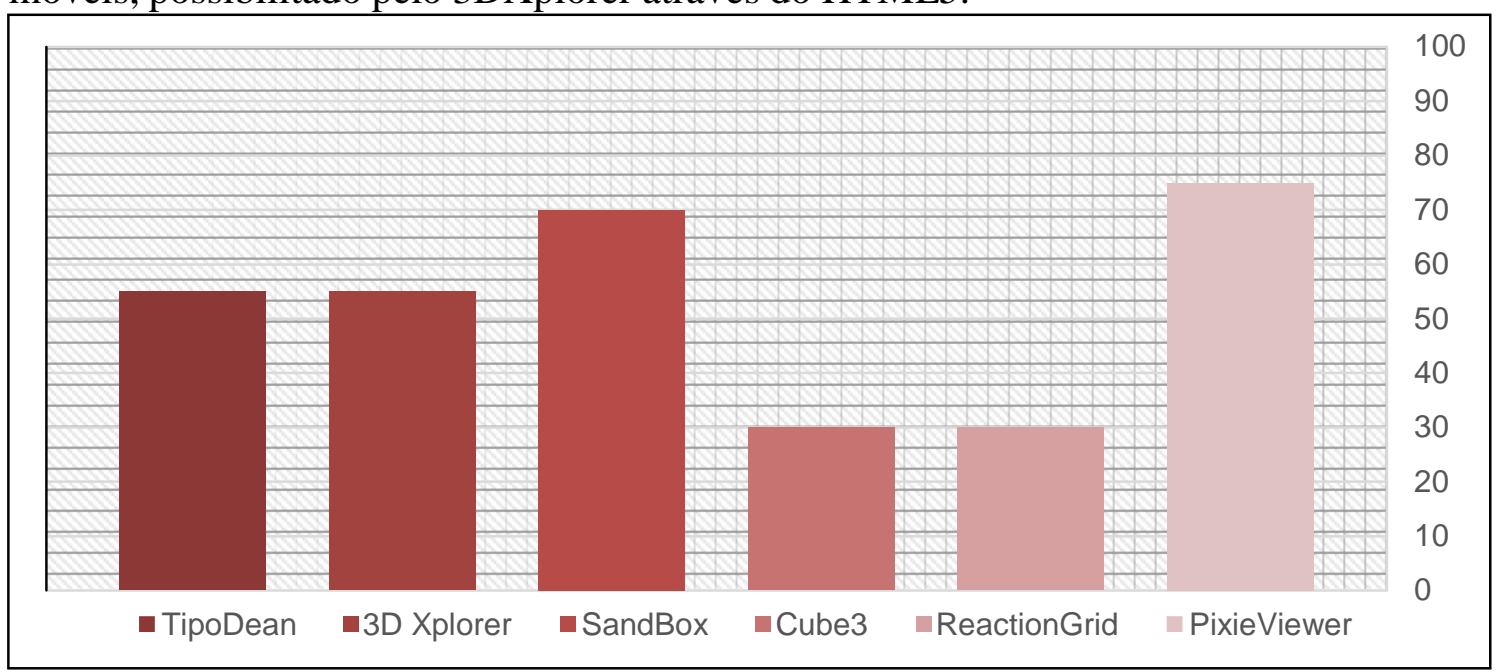

Gráfico 1. Pontuação dos Web Viewers

Os viewers Cube3 e ReactionGrid apresentaram a menor pontuação (30). Isso reflete suas limitações, pois não oferecem a maioria das funcionalidades encontradas em outros visualizadores, como serviços adicionais e acesso por dispositivos móveis. Possuem menor portabilidade, além de possuir uma visualização de menor qualidade.

Por meio da análise e das comparações realizadas neste estudo destacam-se a portabilidade, refletida principalmente nos viewers SandBox e PixieViewer, que não requerem instalações de plugins adicionais. O 3DXplorer, por permitir o acesso a dispositivos móveis através do HTML5. Além da manipulação de objetos, como a importação e a modificação encontradas nos viewers SandBox e PixieViewer. Todos os visualizadores analisados possuem regiões de livre acesso para teste e demonstram facilidade de navegação em pelo menos um dos browsers utilizados neste estudo, apesar de apresentarem algumas limitações de visualização e interação com o cenário.

Como limitação, alguns dos visualizadores testados (Tipodean, ReactionGrid e Cube3) não permitiram o acesso via dispositivos móveis, tornando-se uma desvantagem e sabendo que os dispositivos móveis estão em todos os lugares, essa característica se torna fundamental, visto que a sociedade está cada vez mais conectada e busca as 
facilidades de acesso, considerando que até a metade de 2013 a internet móvel alcançou 77,4 milhões de acesso (Voss et al., 2013).

Por fim, os web viewers são uma alternativa para instituições educacionais que possuem infraestrutura reduzida e não podem hospedar viewers nos seus laboratórios ou servidores. Para utilizar essa tecnologia é necessário apenas de acesso à internet. Além disso, otimizam-se as conexões de rede e as interligações geográficas, no momento em que os MVs e os visualizadores são disponibilizados na rede.

\section{CONCLUSÃO}

A utilização de MV na educação está se tornando uma nova proposta metodológica de auxílio à construção do conhecimento. Porém, devido às limitações que o sistema educacional enfrenta, com relação a disponibilização de recursos tecnológicos, há a necessidade de se buscar alternativas que facilitem a inclusão dos metaversos nesse ambiente. Nesse sentido, os visualizadores web estão entre as opções para a redução das exigências de recursos tecnológicos por parte do usuário, permitindo a utilização de MV no meio educativo, não só por serem disponibilizados na internet, mas também por serem de fácil utilização, exigindo pouco ou nenhum tipo de configuração adicional para o seu funcionamento.

Este estudo teve por objetivo analisar e comparar os web viewers, apresentando uma visão mais ampla e funcional dessas tecnologias. A pesquisa permitiu identificar características essenciais de visualizadores web e sua principal vantagem, a utilização sem a necessidade de instalação ou configuração de hardware e software, bem como a possibilidade de acesso por dispositivos móveis. É importante ressaltar que, ao buscar uma nova alternativa aos viewers, está procurando-se uma maneira mais acessível de introduzir essa tecnologia em ascensão no contexto educativo, visando apoiar a aprendizagem mediada por computador e otimizar o processo ensino-aprendizagem.

Segundo os testes realizados neste estudo os web viewers PixieViewer e SandBox foram considerados os mais completos, recebendo melhor pontuação em suas análises (75 e 70). Isso se deve ao fato de apresentarem a maioria das funcionalidades levantadas na análise. O 3DXplorer (55) destacou-se pelo fácil acesso via dispositivos móveis, e o Tipodean (55) pelo serviço de conversão de mundos virtuais em versão para web. Os viewers Cube3 e ReactionGrid alcançaram a mesma pontuação (30), sendo a menor apresentada na pesquisa, por serem visualizadores mais limitados, principalmente pelo difícil acesso no browser IE e nos dispositivos móveis, por causa do uso do plugin Unity 3D.

Outro aspecto constatado é que das vinte ferramentas analisadas, somente seis foram selecionadas para compor este estudo, as demais foram desclassificadas por não atenderem aos requisitos mínimos elencados. Dentre estas ferramentas, analisou-se o visualizador do OpenWonderland, sendo possível apurar que os problemas elencados nesta pesquisa e de Nunes et al. (2013) não ocorrem, pois o acesso aos conteúdos do ambiente imersivo dá-se por meio de uma ferramenta própria, denominada cliente. Seu funcionamento baseia-se na arquitetura cliente-servidor, onde o cliente é disponibilizado ao usuário através de uma página web utilizando a tecnologia Java Web Start.

Como trabalhos futuros, esta pesquisa pretende estudar outros web viewers específicos para dispositivos móveis. Além disso, pretende-se analisar os viewers web de forma mais abrangente, atingindo todos os atributos que os visualizadores convencionais oferecem. 


\section{REFERÊNCIAS BIBLIOGRÁFICAS}

3DXPLORER. Disponível em <http://www.3dxplorer.com>. Acesso: ago./2014.

ALBUQUERQUE, A. L. P.; VELHO, L.. Togetherness through virtual worlds: how real can be that presence?. In: Puc-Rio Inf. Mcc 48/03, Novembro, 2003.

AMARAL; É.; ÁVILA, B. G.; TAROUCO, L. M. R. Aspectos teóricos e práticos da implantação de um laboratório virtual no opensim. XXIII SBIE, 2012.

ÁVILA, B.; AMARAL, E. M. H.; TAROUCO, L. Implementação de laboratórios virtuais no metaverso opensim. RENOTE. v. 11, $n^{\circ} .1 .2013$.

CUBE3. Disponível em <http://www.cube3.com>. Acesso: 21 de ago. 2014.

FAROOQ, U.; GLAUERT, J. Scalable and consistent virtual worlds: an extension to the architecture of opensimulator. ICCNIT, 2011.

HERPICH, F., JARDIM, R. R., NUNES, F. B., VOSS, G. B., FONTOURA, L. M., MEDINA, R. D. Laboratório Virtual: uma ferramenta imersiva para auxilio no ensino de engenharia de software. XVI SVR, 2014.

HYPERGRID BUSINESS. Disponível em <http://goo.gl/oqhAEl>. Acesso: ago./2014. LIKERT, R. A technique for the measurerement of attiudes. Archives of Psychology, v. 22, n. 140, p. 1-55, 1932.

MEDINA, R. D. (2004). ASTERIX: Aprendizagem significativa e tecnologias aplicadas no ensino de redes de computadores: integrando e explorando possibilidades. Tese de Doutorado. UFRGS, Porto Alegre, 174 páginas.

NÁJERA. A. B. U.; HUESCA, R. R.; MEDINA, M. A.; PULIDO, L. F.; DOMÍNGUEZ, E. L. Percepción de estudiantes universitarios del aprendizaje soportado por mundos virtuales. TISE. 2011. p. 34-41.

NELSON, B. C.; ERLANDSON, B. E. Design for learning in virtual worlds. New York: Routledge - Taylor \& Francis Group. p. 219, 2012.

NUNES, F. B.; VOSS, G. B.; HERPICH, F.; MÜHLBEIER, A. R. K.; POSSOBOM, C. C.; MEDINA, R. D.. Viewers para ambientes virtuais imersivos: uma análise comparativa teórico-prática. RENOTE. v.11 nº 1, 2013.

OPENSIM. Disponível em <http://goo.gl/0Lt6VX>. Acesso: ago./2014.

OPENWONDERLAND. Disponível:<http://openwonderland.org> . Acesso: ago./2014. ORGAZ, G. B., R-Moreno, M. D., Camacho, D., Barrero, D. F.. Clustering avatars behaviours from virtual worlds interactions. WI\&C’12, n.4, p.1 - 7, 2012.

REACTIONGRID. Disponível em <http://reactiongrid.com>. Acesso: jul./2014.

REIS, S. C. Dos, PANCIERA,R.J.,GOMES,A.F.,MENEZES, V. P. Da pesquisa à ação: conectando pressupostos teóricos e pedagógicos no desenvolvimento de um jogo de inglês interdisciplinar em 3D. TISE, 2013, p. 113-120.

SECOND LIFE. Disponível em <http://secondlife.com>. Acesso: ago./2014.

STATCOUNTER GLOBAS STATS. Top 5 desktop, tablet e console browsers from jan to july 2014. Disponível em <http://gs.statcounter.com/> Acesso: jul./2014.

TIPODEAN. Disponível em <http://www.tipodean.com>. Acesso: ago./2014.

VOSS, G. B. TCN5: Teaching Computer Networks In A Free Immersive Virtual

Environment. Dissertação de Mestrado. PPGI - UFSM. 2014.

VOSS, G. B., NUNES, F. B., HERPICH, F., MEDINA, R. D. Ambientes virtuais de aprendizagem e ambientes imersivos: um estudo de caso utilizando tecnologias de computação móvel. XXIV SBIE, 2013.

VW SANDBOX. Disponível em <http://goo.gl/h81FnR>. Acesso: ago./2014. 